\title{
Solvent Reorganization Entropy of Electron Transfer in Polar Solvents
}

\author{
Pradip K. Ghorai and Dmitry V. Matyushov* \\ Department of Chemistry and Biochemistry and the Center for the Early Events in Photosynthesis, Arizona \\ State University, PO Box 871604, Tempe, Arizona 85287-1604
}

Received: October 31, 2005; In Final Form: February 2, 2006

\begin{abstract}
We report the results of molecular dynamics simulations of the solvent reorganization energy of intramolecular electron transfer in a charge-transfer molecule dissolved in water and acetonitrile at varying temperatures. The simulations confirm the prediction of microscopic solvation theories of a positive reorganization entropy in polar solvents. The results of simulations are analyzed in terms of the splitting of the reorganization entropy into the contributions from the solute-solvent interaction and from the alteration of the solvent structure induced by the solute. These two contributions mutually cancel each other, resulting in the reorganization entropy amounting to only a fraction of each component.
\end{abstract}

\section{Introduction}

Like many other properties used to describe chemical reaction dynamics and transport phenomena, the activation barrier of electron transfer (ET) reactions is commonly defined in terms of thermodynamically accessible variables. ET involves two equilibrium states which can generally be characterized by three thermodynamic parameters. The first one, $\Delta F_{0}$, is the difference in free energy between the final and initial electronic states of the donor-acceptor complex immersed in a solvent. Two others are related to small fluctuations around the equilibrium, which can also be described by statistical mechanics. ${ }^{1,2}$ The energy gap between the acceptor and donor electronic states makes the collective reaction coordinate for $\mathrm{ET}^{3-5}$

$$
X=\Delta E\left(q_{1}, \ldots, q_{n}\right)
$$

which depends on a manifold of nuclear coordinates $q_{1}, \ldots, q_{n}$. Two second cumulants $\left\langle(\delta X)^{2}\right\rangle_{i}$, calculated on statistical equilibrium distributions corresponding to the initial $(i=1)$ and final $(i=2)$ states of the ET system, are two other thermodynamic parameters fundamentally accessible for ET reactions. They define the two solvent reorganization energies (classical nuclear motions)

$$
\lambda_{\mathrm{s} i}=\beta\left\langle(\delta X)^{2}\right\rangle_{i} / 2
$$

where $\delta X=X-\langle X\rangle_{i}$ and $\beta=1 / k_{\mathrm{B}} T$.

The Marcus - Hush theory assumes ${ }^{2}$

$$
\lambda_{\mathrm{s}}=\lambda_{\mathrm{s} 1}=\lambda_{\mathrm{s} 2}
$$

The free energy surfaces along the reaction coordinate $X$ are then two intersecting parabolas. This assumption, which is equivalent to the linear response approximation in statistical mechanics calculations, reduces the number of independent thermodynamic parameters to two. Computer simulations of solutes with permanent partial charges immersed in molecular solvents reported previously ${ }^{6-9}$ and presented here (see below) support this assumption. Note, however, that some simulations

* Author to whom correspondence should be addressed. E-mail: dmitrym@asu.edu. with highly charged ions ${ }^{10-12}$ and small optical dyes ${ }^{13}$ result in $\lambda_{\mathrm{s} 1} \neq \lambda_{\mathrm{s} 2}$. All three thermodynamic parameters, $\Delta F_{0}, \lambda_{\mathrm{s} 1}$, and $\lambda_{\mathrm{s} 2}$, can be maintained in a three-parameter model of ET. The requirement $\lambda_{\mathrm{s} 1} \neq \lambda_{\mathrm{s} 2}$ results in nonparabolic free energy surfaces $^{14,15}$ which have been obtained in simulations of polarizable solutes with polarizability changing with electronic transition. ${ }^{16-18}$

The classical nuclear reorganization energy takes a central role in all formulations of the ET theory since it allows one to build the global, nonequilibrium free energy surfaces based on thermodynamic observables obtained for two equilibrium configurations (just two points on the free energy surfaces). The result is a powerful theory that describes both the activated barrier crossing kinetics and optical charge-transfer bands. ${ }^{19,20}$ In view of its central role, the knowledge of the thermodynamics of nuclear reorganization, in particular solvent reorganization, is critical for understanding and interpreting the ET experiment. ${ }^{21}$ The present paper focuses on the solvent reorganization energy, $\lambda_{\mathrm{s}}$.

Experimentally, $\lambda_{\mathrm{s}}$ can be extracted from solvent-induced broadening of vibronic transition lines. This task requires in most cases theoretical modeling of the optical band shape made of many vibronic transitions. In practice, therefore, the reorganization energy is often obtained from the first spectral moments of absorption and emission bands using another relation following from the approximation of two equal-curvature parabolas

$$
\Delta u_{\mathrm{st}}=\hbar\left[\Delta \omega_{\mathrm{st}}-\Delta \omega_{\mathrm{v}}\right]=2 \lambda_{\mathrm{s}}
$$

In this equation, $\Delta \omega_{\text {st }}$ is the difference between the first spectral moments for absorption and emission transitions (Stokes shift) and $\Delta \omega_{\mathrm{v}}$ is the part of the Stokes shift arising from intramolecular vibrations of the solute. The latter is normally obtained from spectroscopic measurements in nonpolar solvents. ${ }^{22}$

Most of what we know theoretically about the thermodynamics of solvent reorganization comes from dielectric continuum models of solvation. ${ }^{2,23-25}$ These models predict that all the information required to describe the variation of $\lambda_{\mathrm{s}}$ with changing external parameters is concentrated in the Pekar factor used for ion solvation and long-distance ET 


$$
c_{0}=\epsilon_{\infty}^{-1}-\epsilon_{\mathrm{s}}^{-1}
$$

or in its Lippert-Mataga analogue used for dipole solvation and intramolecular ET

$$
f_{\mathrm{p}}=\frac{\epsilon_{\mathrm{s}}-1}{2 \epsilon_{\mathrm{s}}+1}-\frac{\epsilon_{\infty}-1}{2 \epsilon_{\infty}+1}
$$

In eqs 5 and $6, \epsilon_{\infty}$ and $\epsilon_{\mathrm{s}}$ are the high-frequency and static dielectric constants of the solvent, respectively. Solvation of nonspherical solutes results in a more complex dependence on the two dielectric constants. ${ }^{25}$ This dependence is normally intermediate between the ionic (eq 5) and dipolar (eq 6) limiting cases. A number of experimental reports ${ }^{26,27}$ confirm the approximately linear trend

$$
\lambda_{\mathrm{s}} \propto c_{0}
$$

predicted by the Marcus theory of ET. ${ }^{2}$ However, there are some recent theoretical calculations $8,9,13,18,28-33$ and laboratory experiments ${ }^{21,22,34,35}$ questioning the universality of eq 7 .

Early microscopic (molecular) theories of solvent reorganization predicted $^{28}$ that the reorganization entropy at constant pressure

$$
S_{P}=-\left(\partial \lambda_{\mathrm{s}} / \partial T\right)_{P}
$$

is positive:

$$
S_{P}>0
$$

This result comes in contradiction to eq 7 when the temperature dependence of two dielectric constants, $\epsilon_{\infty}(T)$ and $\epsilon_{\mathrm{s}}(T)$, is used to calculate $S_{P}$. Equation 7 results in $S_{P}<0$ for strongly polar solvents and in $S_{P}>0$ for weakly polar solvents. Later refinements of the analytical theory ${ }^{9,31,33}$ as well as laboratory experiments $21,26,31,34,36-42$ have confirmed the positive sign of the reorganization entropy (eq 9). This issue is, however, not fully resolved as there is a limited number of experiments reporting $S_{P}<0$ in strongly polar solvents. ${ }^{43-45}$ Note that all data resulting in $S_{P}<0$ have been obtained for charged donoracceptor complexes, while a recent study ${ }^{46}$ indicates that association of charged donor-acceptor complexes with counterions in solution may reverse the sign of the reorganization entropy.

Although there have been many simulations of the ET free energy surfaces, ${ }^{6,7,10-12,47-49}$ the problem of reorganization entropy has never been comprehensively studied by computer simulations. Recent simulations in ref 50 are consistent with eq 9 , but simulation sampling was insufficient and conclusions are mostly qualitative. The aim of this paper is to fill this gap and to present a thermodynamic analysis of the reorganization entropy based on molecular dynamics (MD) simulations.

Many properties significant for the analysis of the experimental reaction rates are left out from our analysis. Most importantly, the force-field solvents employed in the simulations are nonpolarizable. The polarizability effects are significant, in particular in regard to the recent reports of the overestimated dependence of $\lambda_{\mathrm{s}}$ on $\epsilon_{\infty}$ in eq 7.13,18,51 The solute is also nonpolarizable and rigid. Polarizability ${ }^{16-18}$ and flexibility ${ }^{52,53}$ of the solute, which in principle can be incorporated in computer simulations, are still computationally expensive when the entropy of reorganization is concerned. Our analysis is therefore limited to the thermodynamics of interactions between partial charges of the solute and the solvent.

\section{Thermodynamics of Solvent Reorganization}

Consider a solute interacting with the solvent with an additive potential which can be represented by a sum of interaction with each solvent molecule $j=1, \ldots, N$ :

$$
U_{0 \mathrm{~s}}=\sum_{j=1}^{N} v_{0 \mathrm{~s}}(j)
$$

Here " 0 " and "s" refer to the solute and solvent, respectively. The interaction energy $v_{0 \mathrm{~s}}(j)$ depends on the position and orientation of the solvent molecule denoted by " $j$ " in the brackets.

The chemical potential of solvation can be obtained from the thermodynamic $\lambda$-integration ${ }^{54}$

$$
\mu_{0 \mathrm{~s}}=\rho \int_{0}^{1} \mathrm{~d} \lambda \int v_{0 \mathrm{~s}}(1) g_{0 \mathrm{~s}}\left(\lambda v_{0 \mathrm{~s}} ; 1\right) \mathrm{d} \Gamma_{1}
$$

In addition, the average solute-solvent interaction energy is

$$
e_{0 \mathrm{~s}}=\rho \int v_{0 \mathrm{~s}}(1) g_{0 \mathrm{~s}}\left(v_{0 \mathrm{~s}} ; 1\right) \mathrm{d} \Gamma_{1}
$$

Here, $g_{0 \mathrm{~s}}\left(\lambda v_{0 \mathrm{~s}} ; 1\right)$ denotes the solute-solvent distribution function defined on the scaled solute-solvent interaction potential, $\lambda v_{0 \mathrm{~s}^{-}}$ (1), and $\mathrm{d} \Gamma_{1}$ denotes the integration over the phase space of the solvent including the solvent positions and orientations; $\rho$ is the solvent number density. The linear response approximation assumes that the pair distribution function $g_{0 \mathrm{~s}}\left(\lambda v_{0} ; 1\right)$ can be linearly expanded in the potential $\lambda v_{0 \mathrm{~s}}(1)$ relative to a reference state which is independent of the solute-solvent interaction and is thus independent of solvent orientations: ${ }^{54}$

$$
g_{0 \mathrm{~s}}\left(\lambda v_{0 \mathrm{~s}} ; 1\right)=g_{0 \mathrm{~s}}^{(0)}\left(\mathbf{r}_{1}\right)+\lambda g_{0 \mathrm{~s}}^{(1)}(1)
$$

where $g_{0 \mathrm{~s}}^{(0)}\left(\mathbf{r}_{1}\right)$ is the reference distribution function and

$$
\begin{aligned}
& g_{0 \mathrm{~s}}^{(1)}(1)=-\beta g_{0 \mathrm{~s}}^{(0)}\left(\mathbf{r}_{1}\right)\left(v_{0 \mathrm{~s}}(1)-\left\langle U_{0 \mathrm{~s}}\right\rangle\right)- \\
& \quad \beta \rho \int v_{0 \mathrm{~s}}(2) g_{0 \mathrm{~s}}^{(0)}(12) \mathrm{d} \Gamma_{2}
\end{aligned}
$$

In eq 14 , the average $\langle\ldots\rangle$ is performed over the reference distribution function, e.g.

$$
\left\langle U_{0 \mathrm{~s}}\right\rangle=\rho \int g_{0 \mathrm{~s}}^{(0)}\left(\mathbf{r}_{1}\right) v_{0 \mathrm{~s}}(1) \mathrm{d} \Gamma_{1}
$$

Further, $g_{0 \text { s }}{ }^{(0)}(12)$ in eq 14 is the three-particle, solute-solventsolvent distribution function of the reference system.

By substituting eqs 13 and 14 into eqs 11 and 12, one gets

$$
\begin{gathered}
\mu_{0 \mathrm{~s}}=\left\langle U_{0 \mathrm{~s}}\right\rangle-(\beta / 2)\left\langle\left(\delta U_{0 \mathrm{~s}}\right)^{2}\right\rangle \\
e_{0 \mathrm{~s}}=\left\langle U_{0 \mathrm{~s}}\right\rangle-\beta\left\langle\left(\delta U_{0 \mathrm{~s}}\right)^{2}\right\rangle
\end{gathered}
$$

where $\delta U_{0 \mathrm{~s}}=U_{0 \mathrm{~s}}-\left\langle U_{0 \mathrm{~s}}\right\rangle$. When $\left\langle U_{0 \mathrm{~s}}\right\rangle=0$, eq 16 yields

$$
\mu_{0 \mathrm{~s}}=e_{0 \mathrm{~s}} / 2=-(\beta / 2)\left\langle\left(\delta U_{0 \mathrm{~s}}\right)^{2}\right\rangle
$$

The thermodynamics of the solvent reorganization can be understood from the above arguments by considering a fictitious solute with the geometry of the real solute, but the charge distribution obtained as a difference of atomic charges in the final and initial charge-transfer states, $\Delta q_{j}=q_{2 j}-q_{1 j}$ (no polarizability change is considered here). The solute-solvent 
interaction potential is then the difference of interaction potentials in the final and initial states, $\Delta v_{0 \mathrm{~s}}(1)=v_{0 \mathrm{~s}, 2}(1)-$ $v_{0 \mathrm{~s}, 1}(1), \Delta U_{0 \mathrm{~s}}=\sum_{j} \Delta v_{0 \mathrm{~s}}(j)$. Once the donor-acceptor energy gap $X=\Delta E$ in eq 1 is related to $\Delta U_{0 \text { s }}$ by a constant shift, eq 17 leads to the following relation (see also ref 8 ):

$$
\lambda_{\mathrm{s}}=-\mu_{0 \mathrm{~s}}=-e_{0 \mathrm{~s}} / 2=(\beta / 2)\left\langle\left(\delta \Delta U_{0 \mathrm{~s}}\right)^{2}\right\rangle
$$

where now $\mu_{0 \mathrm{~s}}$ and $e_{0 \mathrm{~s}}$ refer to solvation of the fictitious solute with the solute-solvent interaction energy $\Delta U_{0 \mathrm{~s}}$.

From eq 18, the solvent reorganization (free) energy can be split into the energy and constant-volume entropy components

$$
\lambda_{\mathrm{s}}=E-T S_{V}
$$

where

$$
E=2 \lambda_{\mathrm{s}}+\Phi
$$

and

$$
S_{V}=\lambda_{\mathrm{s}} / T+\Phi / T
$$

In eqs 20 and 21 , the components including $\lambda_{\mathrm{s}}$ refer to the statistics of solute-solvent interactions, while $\Phi$ reflects the components of the entropy and internal energy which arise from the alteration of the solvent-solvent structure induced by the solute. ${ }^{55-57}$ The component $\Phi$ does not affect the solvation chemical potential as it identically cancels out in eq 19. However, it can significantly affect the observed reorganization entropy obtained as the temperature derivative of the reorganization energy

$$
S_{V}=-\left(\partial \lambda_{\mathrm{s}} / \partial T\right)_{V}
$$

The constant-pressure entropy $S_{P}$ differs from $S_{V}$ by a term containing the derivative of $\lambda_{\mathrm{s}}$ over density:

$$
S_{P}=S_{V}+\rho \alpha_{p}\left(\frac{\partial \lambda_{\mathrm{s}}}{\partial \rho}\right)_{T}
$$

where $\alpha_{p}$ is the constant-pressure expansivity of the solvent.

One can calculate $\Phi$ by noting that the variance of the potential difference $\Delta U_{0 \mathrm{~s}}$ can be connected to $\lambda_{\mathrm{s}}$ by the following relation:

$$
2 k_{\mathrm{B}} T \lambda_{\mathrm{s}}=\frac{\int\left(\delta \Delta U_{0 \mathrm{~s}}\right)^{2} \mathrm{e}^{-\beta \mathrm{H}_{0}} \mathrm{~d} \Gamma}{\int \mathrm{e}^{-\beta \mathrm{H}_{0}} \mathrm{~d} \Gamma}
$$

where $H_{0}$ is the Hamiltonian of the system when the solutesolvent electrostatic potential $\Delta U_{0 \mathrm{~s}}$ is switched off; $\mathrm{d} \Gamma=\mathrm{d} \Gamma_{1}$ $\ldots \mathrm{d} \Gamma_{\mathrm{N}}$. The Hamiltonian $H_{0}$ thus includes all the solventsolvent interactions and nonpolar solute-solvent interactions (Lennard-Jones (LJ) potential in our simulations). Differentiation of 24 over temperature yields

$$
\Phi=-\left(\beta^{2} / 2\right)\left\langle\left(\delta \Delta U_{0 \mathrm{~s}}\right)^{2} \delta H_{0}\right\rangle
$$

where $\delta H_{0}=H_{0}-\left\langle H_{0}\right\rangle$.

The term "solvent reorganization energy" has been used in solvation literature to describe the components of the solvation energy and entropy arising from the alteration of the solvent structure induced by the solute. ${ }^{55,58}$ This energy is distinct from the ET reorganization energy considered here. Further, the analogue of the energy $\Phi$ appearing in the solvation energy is a second-order correlator, ${ }^{5-57}$ in contrast to the third-order correlator for $\Phi$ in eq 25 . In order to distinguish $\Phi$ from from its analogue in solvation theories, we will call $\Phi$ the energy of solvent restructuring.

The contribution of solvent restructuring to the ET reorganization entropy is represented by correlated fluctuations of the solute-solvent electrostatic potential and of the Hamiltonian $H_{0}$, the main part of which is the solvent-solvent interaction energy. This result clarifies the fundamental origin of the failure of models neglecting the solvent structure ${ }^{2,23-25}$ to describe the entropy of solvation: ${ }^{31}$ the problem of solvent structure alteration is simply not addressed by such models.

How can we relate the reorganization energies and Stokes shifts obtained in the laboratory and/or computer experiment to the linear response thermodynamics considered here? The equality of reorganization energies in eq 3 implies independence of the second cumulant of $\Delta U_{0 \mathrm{~s}}$ of the electronic state of the solute. ${ }^{47}$ This result can be obtained by zero-order expansion of the solute-solvent distribution function replacing the actual distribution function $g_{0 \mathrm{~s}}\left(v_{0 \mathrm{~s}, i}, 1\right)$ with the reference distribution function $g_{0 \mathrm{~s}}^{(0)}\left(\mathbf{r}_{1}\right)$ independent of the solute-solvent electrostatic potential. The situation is different for the Stokes shift. The Stokes shift

$$
\Delta u_{\mathrm{st}}=\rho \int \Delta v_{0 \mathrm{~s}}(1)\left[g_{0 \mathrm{~s}}\left(v_{0 \mathrm{~s}, 1}, 1\right)-g_{0 \mathrm{~s}}\left(v_{0 \mathrm{~s}, 2}, 1\right)\right] \mathrm{d} \Gamma_{1}
$$

is determined by the alteration of the solute-solvent distribution function caused by the transition, where $g_{0 \mathrm{~s}}\left(v_{0 \mathrm{~s}, i}, 1\right)$ refers to the distribution function characterized by the potential $v_{0 \mathrm{~s}, i}$. If the linear expansion, given by eqs 13 and 14, is used for each of $g_{0 \mathrm{~s}}\left(v_{0 \mathrm{~s}, i}, 1\right)$, one gets the linear response result in eq 4 . Despite the fact that both eq 3 and eq 4 are based on the same linear response approximation, the equality of the reorganization energies comes from the zero-order expansion and the connection between the Stokes shift and the reorganization energy comes from approximating the difference of distribution functions by the linear expansion term. The robustness of these two approximations may differ in real systems as our simulations actually indicate.

\section{Model and Simulation Protocol}

The solute in our simulations was represented by the $p$-nitroaniline molecule. Charge transfer in $p$-nitroaniline results in a dipole moment change of about 3.7 D. The ground-state geometry and charge distribution were obtained using GAUSSIAN $03^{59}$ (MP2, 6-31+G*). The geometry of $p$-nitroaniline from $\mathrm{X}$-ray experiments ${ }^{60}$ was used as a starting model for the geometry optimization. The ground-state geometry was used to calculate the ground-state and excited-state (CIS) atomic charges by fitting the electrostatic potential constrained to reproduce the dipole moment $(\mathrm{Pop}=$ Dipole in GAUSSIAN 03). The ground-state, $7.18 \mathrm{D}$, and excited-state, $10.88 \mathrm{D}$, dipoles conform well to the results reported in the literature. ${ }^{61,62}$ The calculated atomic charges are used in a 16-site model of $p$-nitroaniline which includes long-range Coulomb interactions with partial charges of the solvent and short-range LJ $(6-12)$ interactions. The LJ parameters of the solute atoms are based on the OPLS parametrization, ${ }^{63}$ and the solute-solvent cross interactions are obtained from the Lorentz-Berthelot combination rules. ${ }^{64}$

Two solvents, water and acetonitrile, have been used in MD simulations employing the DL_POLY package. ${ }^{65}$ The simple point-charge (SPC/E) model ${ }^{66}$ was used for water, and the potential parameters by Bohm et al. ${ }^{67}$ were used for acetonitrile. 

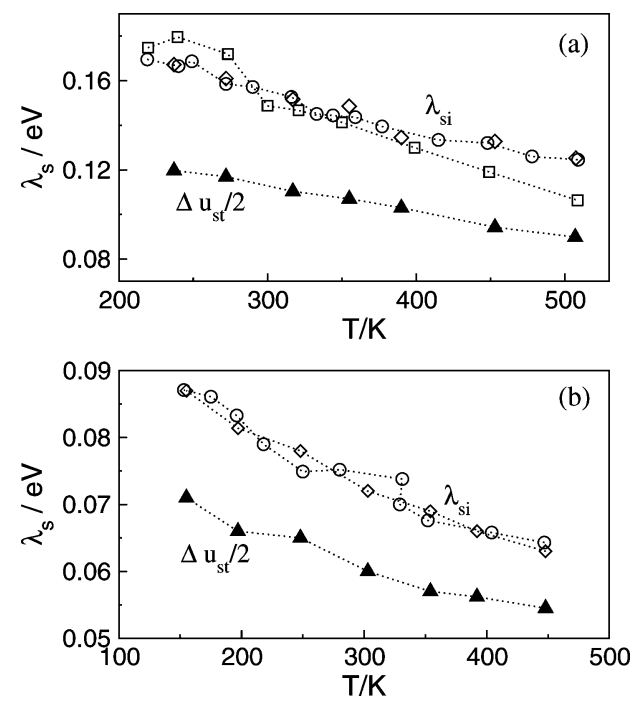

Figure 1. Temperature dependence of the solvent reorganization energy of $p$-nitroaniline in SPC/E water (a) and acetonitrile (b) at constant volume (circles and diamonds) and at constant pressure (squares). Circles and squares refer to the solute ground state $\left(\lambda_{\mathrm{s} 1}\right)$, whereas diamonds refer to the solute charge-transfer state $\left(\lambda_{\mathrm{s} 2}\right)$. Triangles refer to half of the Stokes shift, $\Delta u_{\mathrm{s}} / 2$. The dotted lines connect the simulation points.

Acetonitrile is assumed to be rigid with parameters corresponding to the equilibrium bond lengths and angles ${ }^{68}$ of an isolated molecule.

A time step of $1.0 \mathrm{fs}$ was used for MD integration, which yielded good conservation of linear momentum and energy (fluctuations of energy below $10^{-4} \mathrm{~kJ} / \mathrm{mol}$ ). $\mathrm{NVE}^{64}$ simulations in water have been carried out in a cubic cell with the side length of $24.075 \AA$ containing 466 water molecules and one $p$ nitroaniline molecule. NVE simulations in acetonitrile were done in a cubic cell with the side length of $27.99 \AA$ with 250 acetonitrile molecules and one $p$-nitroaniline molecule. In both cases, the simulation box is chosen to reproduce the solvent density at ambient conditions, $\rho=0.997 \mathrm{~g} / \mathrm{cm}^{3}$ for water and $\rho=0.782 \mathrm{~g} / \mathrm{cm}^{3}$ for acetonitrile. The cutoff radius for $\mathrm{LJ}$ interactions is equal to half of the box length. The Coulomb interactions are treated by the Ewald summation method ${ }^{69}$ which splits the sum over the periodic images of the simulation cell into a damped real space sum and a reciprocal space sum. ${ }^{70}$ Summation in real space is truncated at $L / 2$ (convergence parameters are $0.2346 \AA^{-1}$ for water and $0.2713 \AA^{-1}$ for acetonitrile). Summation in reciprocal space involves approximately 2300 vectors with their magnitudes limited by 5.0 $\AA^{-1}$ for water and by $9.0 \AA^{-1}$ for acetonitrile. Equilibrium averages were calculated from configurations stored at $0.1 \mathrm{ps}$ intervals. For solvation in water, equilibration time was 400 ps and production time was $1.0 \mathrm{~ns}$. For solvation in acetonitrile, equilibration was $500 \mathrm{ps}$ long and production runs were $2.0 \mathrm{~ns}$ long at temperatures above $372 \mathrm{~K}$. At lower temperatures, the equilibration and production time were $800 \mathrm{ps}$ and $4.0 \mathrm{~ns}$, respectively. We have also performed NPT MD simulations of $p$-nitroaniline in water at $300 \mathrm{~K}$ and varying pressure with the same simulation setup as for NVE simulations.

\section{Results and Discussion}

The main result of our simulations is the confirmation of the earlier theoretical ${ }^{8,28,31}$ and experimental ${ }^{21,26,31,34,36-40,42}$ findings of the positive value of the reorganization entropy (negative slope of the reorganization energy with increasing temperature, Figure 1) both at constant-volume and constant-pressure condi-
TABLE 1: Entropies of Reorganization at $300 \mathrm{~K}$

\begin{tabular}{llccccc}
\hline source of data & \multicolumn{1}{c}{ solvent } & $y$ & $\lambda_{\mathrm{s}}, \mathrm{eV}$ & $S_{V} / k_{\mathrm{B}}$ & $S_{P} / k_{\mathrm{B}}$ & $T S_{P} / \lambda_{\mathrm{s}}$ \\
\hline $\mathrm{MD}$ & $\mathrm{SPC} / \mathrm{E}$ water & $6.4^{a}$ & 0.15 & 1.95 & 3.0 & 0.51 \\
$\mathrm{MD}$ & $\mathrm{ACN}$ & $6.54^{a}$ & 0.07 & 1.03 & & \\
experiment $^{b}$ & $\mathrm{ACN}^{c}$ & $10.7^{d}$ & 0.62 & & 9.0 & 0.38 \\
experiment & $\mathrm{ACN}^{e}$ & 10.7 & 0.56 & & 4.5 & 0.21 \\
experiment & diethyl ether $^{c}$ & 0.58 & 0.34 & & 7.6 & 0.57 \\
experiment & tetrahydrofuran $^{c}$ & 1.31 & 0.50 & & 21.1 & 1.08
\end{tabular}

${ }^{a} y=(4 \pi / 9) \beta \rho m^{2}$, calculated from dipole moments $m=2.35 \mathrm{D}$ and $m=4.146 \mathrm{D}$ for force-field water and acetonitrile, respectively; "ACN" refers to acetonitrile. ${ }^{b}$ Experimental results refer to constant-pressure conditions. ${ }^{c}$ From temperature dependence of the Stokes shift. ${ }^{34}$ ${ }^{d}$ Calculated with account for polarizability effects of the solvent according to procedure described in ref $18 .{ }^{e}$ From resonance Raman measurements of optical band shapes of betaine- $30 .{ }^{40}$

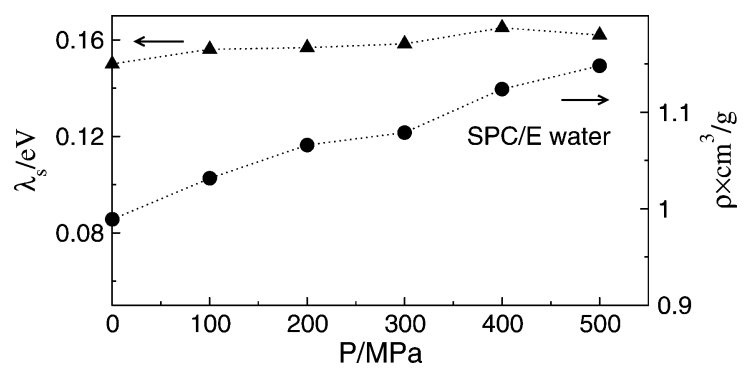

Figure 2. $\lambda_{\mathrm{s}}$ (triangles) and density (circles) of SPC/E water vs pressure from NPT MD simulations at $T=300 \mathrm{~K}$. The dashed lines connect the simulation points.

tions (Table 1). We have also performed simulations of $\lambda_{\mathrm{s}}$ in water at constant temperature of $T=300 \mathrm{~K}$ and varying pressure (NPT MD). Figure 2 shows $\lambda_{\mathrm{s}}(P)$ along with the water density $\rho(P)$. These data are consistent with the notion that $S_{P}>S_{V}$ at $T=300 \mathrm{~K}$ since

$$
S_{P}-S_{V}=\frac{\alpha_{p}}{\beta_{T}}\left(\frac{\partial \lambda_{\mathrm{s}}}{\partial P}\right)_{T}
$$

with $\alpha_{p}>0$ and $\left(\partial \lambda_{\mathrm{s}} / \partial P\right)_{T}>0$ in our simulations. Based on $\beta_{T}$ $=3.14 \times 10^{-10} \mathrm{~Pa}^{-1}$ from Figure $2\left(4.57 \times 10^{-10} \mathrm{~Pa}^{-1}\right.$ for ambient water) and expansivity of ambient water $\alpha_{\mathrm{p}}=2.6 \times$ $10^{-4} \mathrm{~K}^{-1}$, the difference $S_{P}-S_{V}$ is $0.3 k_{\mathrm{B}}$ from eq 27 . This is about three times smaller than what follows from Table 1 indicating that NPT simulations most likely overestimate the value of $S_{P}$

One of the assumptions made in deriving eqs $18-21$ is the equality to zero of the average $\left\langle\Delta U_{0 s}\right\rangle$ taken with the referencesystem equilibrium distribution. Formally, this relation holds because the average includes integration over the orientations of the solvent molecules. Since $g_{0 \mathrm{~s}}^{(0)}\left(\mathbf{r}_{1}\right)$ is independent of these orientations and the average of the interaction potential over solvent rotations is zero, the overall average is zero too. We have tested the consistency of our analysis by computer simulations. A solute with the full set of LJ interactions but with all partial charges set to zero (reference system) was simulated in water and acetonitrile. The calculated $\left\langle\Delta U_{0 \mathrm{~s}}\right\rangle$ turned out to be equal to $-2.85 \times 10^{-3} \mathrm{eV}$ in water and $-6.31 \times$ $10^{-4} \mathrm{eV}$ in acetonitrile. Both numbers are significantly lower than the reported reorganization energies.

Our simulations confirm the equivalence of the reorganization energies calculated for the charge-separation $\left(\lambda_{\mathrm{s} 1}\right)$ and charge recombination $\left(\lambda_{\mathrm{s} 2}\right)$ reactions (eq 3 , cf. open circles and diamonds in Figure 1). However, half of the Stokes shift (eq 4) is uniformly lower than the reorganization energy, by $30 \%$ in water and by $40 \%$ in acetonitrile at $300 \mathrm{~K}$ (Figure 1). Deviations 


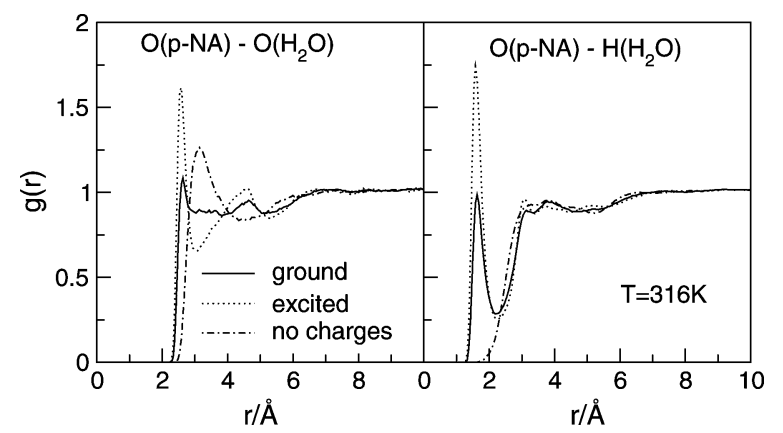

Figure 3. Oxygen-oxygen (left panel) and oxygen-hydrogen (right panel) pair distribution functions between oxygens of $p$-nitroaniline (p-NA) and SPC/E water. The solid lines refer to the charge distribution of $p$-nitroaniline in the ground state, and the dashed lines refer to the excited, charge-transfer state. The dash-dotted lines refer to p-NA in the reference state when all partial charges are set equal to zero. Equilibrium trajectories in all charge states were accumulated from NVE MD runs at $T=316 \mathrm{~K}$.

from the linear response predictions are normally traced back to a strong electrostatic coupling of the solvent molecules to the solute, invalidating the linear perturbation expansion. This is illustrated in Figure 3 where we show the pair distribution functions between the oxygens of $p$-nitroaniline and oxygens and hydrogens of water. The pair distribution function is calculated for the reference solute, which has all LJ interactions of $p$-nitroaniline but no partial charges, and for the ground and excited states of the solute. A significant alteration of the pair distribution function is obvious; in particular the hydrogen peak in the first solvation shell does not appear without a negative charge placed on oxygen.

The difference between the actual distribution function and that of the reference system apparently does not affect the statistics of the electrostatic potential fluctuations produced by the solvent, which are represented by the reorganization energy. This observation attests to the collective nature of electrostatic potential fluctuations in dense polar solvents which might be little affected by local structural changes in the first solvation shell of the solute. The Stokes shift, on the contrary, is sensitive to the local effects since it is defined by the difference of the distribution functions in the excited and ground states (eq 26) which might be less reliably reproduced by the first-order expansion.

Deviations from the linear Stokes shift dynamics in dipolar, nonpolarizable solutes interacting with electrostatic forces with protic solvents were examined by Fonseca and Ladanyi ${ }^{71}$ and later by Geissler and Chandler. ${ }^{72}$ In the former study, considering solvent relaxation to a flip of dipole in a solute diatomic, nonlinear solvation manifested itself in the deviation between the time-dependent Stokes shift and the equilibrium time correlation function of the ET energy gap. The second study ${ }^{72}$ of the same system has shown that the width of the energy gap distribution evolves with time. Solvation nonlinearities in both cases were a result of a strong electrostatic coupling of water protons to the negative side of the solute diatomic. The physical situation observed here is qualitatively similar. It is the change in the population of partially negative oxygens of $p$-nitroaniline that creates Stokes shift deviating from the prediction of linear response. Note that this effect is local and, therefore, cannot be described by models of ET and solvation dynamics considering coupling of the solute electrostatics to a collective solvent mode. Indeed, we are not aware of any existing model ${ }^{2,14,73}$ allowing equal reorganization energies (eq 3 ) and, at the same time, $\Delta u_{\mathrm{st}}$ $\neq 2 \lambda_{\mathrm{s}}$.

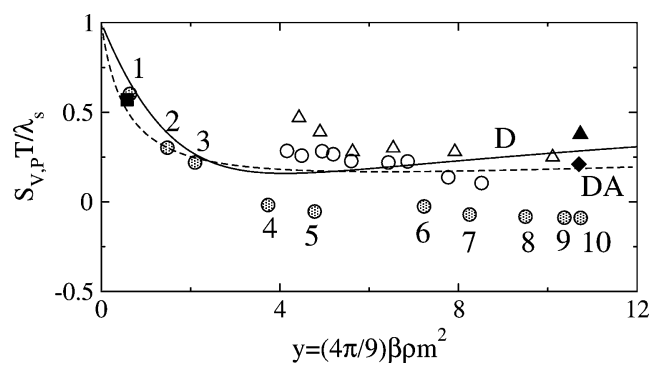

Figure 4. $S_{V, P} T / \lambda_{\mathrm{s}}$ vs $y=(4 \pi / 9) \beta \rho m^{2}$ for $p$-nitroaniline in SPC/E water (open circles) and acetonitrile (open triangles) from NVE MD simulations in which the three-particle correlator in eq 25 was directly calculated from trajectories in equilibrium with the ground-state solute. The dipolar density $y=(4 \pi / 9) \beta \rho m^{2}$ was varied by changing temperature. Also shown are the results of NVT MC simulations for a spherical dipolar solute ${ }^{8}$ (solid line, "D") and a contact diatomic solute with opposite charges ${ }^{9} \mathrm{D}^{+}-\mathrm{A}^{-}$(dashed line, "DA") in dipolar hard-sphere solvents with varying dipole moment. The two lines are obtained by direct differentiation of the simulated reorganization energies as functions of $y$. The closed points correspond to results reported in ref 34 for diethyl ether (closed square) and acetonitrile (closed triangle) and in ref 40 for acetonitrile (closed diamond) (see Table 1). The shaded circles indicate molecular solvents for which entropies and reorganization energies are calculated by using the Lippert-Mataga equation (eqs 6 and 30). The numbers on the plot indicate chloroform (1), tetrahydrofuran (2), 1,1-dichloroethane (3), acetone (4), propionitrile (5), dimethylformamide (6), nitromethane (7), acetonitrile (8), dimethyl sulfoxide (9), and propylene carbonate (10). The shaded circles and closed points refer to the entropy $S_{P}$, while the open points refer to the entropy $S_{V}$.

Despite similarities in the strong coupling between the negative charges of the solute to water protons in our study and in refs 71 and 72 , there are significant quantitative differences in the overall solute-solvent electrostatic coupling. In the diatomic studied in refs 71 and 72 , the flip of the dipole creates the difference dipole moment $\Delta m_{0} \simeq 7.4 \mathrm{D}$ in a small solute of the diameter $\sigma_{0} \simeq 3.8 \AA$. This corresponds to an effective dipole moment $\left(m_{0}^{*}\right)^{2}=\beta\left(\Delta m_{0}\right)^{2} / \sigma_{0}^{3} \simeq 94$. For $p$-nitroaniline studied here, $\Delta m_{0} \simeq 3.7 \mathrm{D}$ and $\sigma_{0} \simeq 6.3 \AA$, resulting in $\left(m^{*}{ }_{0}\right)^{2} \simeq 1.3$. The value of $\left(m^{*}{ }_{0}\right)^{2}$ should be compared to $\left(m^{*}\right)^{2}=\beta m^{2} / \sigma^{3}$ of the solvent, where $m$ is the solvent dipole moment and $\sigma$ is the solvent diameter. Pronounced effects of nonlinear electrostatic solvation are expected when $m^{*}$ and $m^{*}$ are markedly different, creating a disbalance between the solute-solvent and solvent-solvent interactions. ${ }^{8}$ With $\left(m^{*}\right)^{2} \simeq 6.4$ for water, this disbalance is much stronger for the model system studied in refs 71 and 72 than for aqueous $p$-nitroaniline.

In Figure 4 we report the direct calculation of the constantvolume reorganization entropy from eqs 21 and 25 . The threeparticle correlator in eq 25 , responsible for the alteration of the solvent-solvent energetics by the solute, was calculated directly from MD trajectories in equilibrium with the solute in the ground electronic state. We compare these results with NVT Monte Carlo (MC) simulations of a spherical point dipole in fluids of dipolar hard spheres ${ }^{8}$ (line marked " $D$ " in Figure 4) and to NVT $\mathrm{MC}$ simulations of a charge-transfer diatomic, $\mathrm{D}^{+}-\mathrm{A}^{-}$, in dipolar hard sphere liquids 9 (marked "DA" in Figure 4). In order to minimize the effect of the solute size and shape, we consider the reduced parameter

$$
\frac{T S_{V}}{\lambda_{\mathrm{s}}}=1-\beta \frac{\left\langle\left(\delta \Delta U_{0 \mathrm{~s}}\right)^{2} \delta H_{0}\right\rangle}{\left\langle\left(\delta \Delta U_{0 \mathrm{~s}}\right)^{2}\right\rangle}
$$

which directly quantifies the relative effect of the solvent 
structure alteration on the reorganization entropy. In the case of solvation of dipolar and diatomic solutes, the entropy $S_{V}$ was calculated by direct temperature differentiation of the simulation data obtained at different values of dipolar strength

$$
y=(4 \pi / 9) \beta \rho m^{2}
$$

where $m$ is the solvent dipole moment and $\rho$ is the number density. The most significant result of our calculations is the dramatic effect of the solvent structure alteration ( $\Phi$ in eq 21) on the reorganization entropy. The observed entropy is the result of the mutual cancellation of two large numbers with the resultant entropy as small as $15 \%$ of each component in eq 21 .

We have also shown some experimental data in Figure 4. Closed points refer to results of steady-state optical ${ }^{34}$ and resonance $\operatorname{Raman}^{40}$ measurements at different temperatures resulting in reorganization entropies $S_{P}$ listed in Table 1 (the point for tetrahydrofuran falls out of the range of the plot for unclear reasons). These results are compared with the values $T S_{P} / \lambda_{\mathrm{s}}$ calculated for a number of polar solvents using the Lippert-Mataga equation for the nuclear part of dipole solvation

$$
\lambda_{\mathrm{s}} \propto f_{\mathrm{p}}
$$

where $f_{\mathrm{p}}$ is given by eq 6 (the dielectric constants are taken from ref 74). Any dependence on the cavity radius cancels out in the reduced value $T S_{P} / \lambda_{\mathrm{s}}$ thus providing a direct comparison between the simulations and the dielectric continuum approximation. There is a very good match between simulations and dielectric continuum results at low solvent polarities (solvent quadrupolar effects, which may become significant in this range, ${ }^{22,29,33}$ are not considered here). Once the polarity increases, the dielectric continuum approximation predicts the wrong sign for the reorganization entropy.

There is a general agreement between different simulations for the reorganization entropy shown in Figure 4. The thermodynamic state of the liquid of dipolar hard spheres is fully determined by two dimensionless parameters, $\rho \sigma^{3}$ and $y$ (eq 29). Therefore, changing temperature or the dipole moment is equivalent for that fluid once it results in the same value of $y$. The force-field solvents used in the simulations do not share this universality, but the results of changing the temperature in these solvents generally agree with the results of alteration of the dipole moment in dipolar solvents. More experimental and simulation data are required before definite conclusions regarding the universality of the dependence of the entropy on $y$ shown in Figure 4 can be made.

\section{Conclusions}

The direct simulations of the reorganization entropy presented here show its positive sign for electronic transitions in molecules dissolved in dense polar solvents. The entropy splits into the term $\lambda_{\mathrm{s}} / T$ reflecting the statistics of the solute-solvent interactions and the term $\Phi / T$ originating from restructuring of the solvent induced by the solute. These two terms are of opposite sign, with the negative second component almost completely compensating the positive first term. We have observed an unusual deviation from the linear response in which the reorganization energies for the ground and excited states of the solute are equal within simulation uncertainties whereas half of the Stokes shift is $30-40 \%$ below the reorganization energy.

Acknowledgment. This research was supported by the National Science Foundation (CHE-0304694). This is publication \#644 from the ASU Photosynthesis Center.

\section{References and Notes}

(1) Kubo, R.; Toyozawa, Y. Prog. Theor. Phys. 1955, 13, 160.

(2) Marcus, R. A. Rev. Mod. Phys. 1993, 65, 599.

(3) Lax, M. J. Chem. Phys. 1952, 20, 1752.

(4) King, G.; Warshel, A. J. Chem. Phys. 1990, 93, 8682.

(5) Tachiya, M. J. Phys. Chem. 1993, 97, 5911.

(6) Hwang, J.-K.; Warshel, A. J. Am. Chem. Soc. 1987, 109, 715.

(7) Kuharski, R. A.; Bader, J. S.; Chandler, D.; Sprik, M.; Klein, M.

L.; Impey, R. W. J. Chem. Phys. 1988, 89, 3248.

(8) Milischuk, A.; Matyushov, D. V. J. Phys. Chem. A 2002, 106, 2146.

(9) Matyushov, D. V. J. Chem. Phys. 2004, 120, 7532.

(10) Yelle, R. B.; Ichiye, T. J. Phys. Chem. B 1997, 101, 4127.

(11) Hartnig, C.; Koper, M. T. M. J. Chem. Phys. 2001, 115, 8540.

(12) Blumberger, J.; Sprik, M. J. Phys. Chem. B 2005, 109, 6793.

(13) Bader, J. S.; Berne, B. J. J. Chem. Phys. 1996, 104, 1293.

(14) Matyushov, D. V.; Voth, G. A. J. Chem. Phys. 2000, 113, 5413.

(15) Matyushov, D. V.; Voth, G. A. New developments in the theoretical description of charge-transfer reactions in condensed phases. In Reviews in Computational Chemistry, Vol. 18; Lipkowitz, K. B., Boyd, D. B., Eds.; Wiley-VCH: New York, 2002.

(16) Ando, K. J. Chem. Phys. 1997, 107, 4585.

(17) Small, D. W.; Matyushov, D. V.; Voth, G. A. J. Am. Chem. Soc. 2003, 125,7470

(18) Gupta, S.; Matyushov, D. V. J. Phys. Chem. A 2004, 108, 2087.

(19) Barbara, P. F.; Meyer, T. J.; Ratner, M. A. J. Phys. Chem. 1996

100,13148 .

(20) Chen, P.; Meyer, T. J. Chem. Rev. 1998, 98, 1439.

(21) Zimmt, M. B.; Waldeck, D. H. J. Phys. Chem. A 2003, 107, 3580.

(22) Reynolds, L.; Gardecki, J. A.; Frankland, S. J. V.; Maroncelli, M. J. Phys. Chem. 1996, 100, 10337.

(23) German, E. D.; Kuznetsov, A. M. Electrochim. Acta 1981, 26, 1595

(24) Lee, S.; Hynes, J. T. J. Chem. Phys. 1988, 88, 6853.

(25) Brunschwig, B. S.; Ehrenson, S.; Sutin, N. J. Phys. Chem. 1986, $90,3657$.

(26) Grampp, G.; Jaenicke, W. Ber. Bunsen-Ges. Phys. Chem. 1984, 88,335 . This paper does not directly report the slope of the reorganization energy vs temperature. The negative slope can be, however, extracted by combining the dependence of the rate on the Pekar factor with the rate temperature dependence.

(27) Hupp, J. T.; Dong, Y.; Blackbourn, R. L.; Lu, H. J. Phys. Chem. 1993, 97, 3278.

(28) Matyushov, D. V. Chem. Phys. 1993, 174, 199

(29) Perng, B.-C.; Newton, M. D.; Raineri, F. O.; Friedman, H. L. J. Chem. Phys. 1996, 104, 7153.

(30) Perng, B.-C.; Newton, M. D.; Raineri, F. O.; Friedman, H. L. J. Chem. Phys. 1996, 104, 7177.

(31) Vath, P.; Zimmt, M. B.; Matyushov, D. V.; Voth, G. A. J. Phys. Chem. B 1999, 103, 9130.

(32) Raineri, F. O.; Friedman, H. L. Adv. Chem. Phys. 1999, 107, 81.

(33) Milischuk, A. A.; Matyushov, D. V. J. Chem. Phys. 2005, 123, 044501.

(34) Vath, P.; Zimmt, M. B. J. Phys. Chem. A 2000, 104, 2626.

(35) Read, I.; Napper, A.; Zimmt, M. B.; Waldeck, D. H. J. Phys. Chem. A 2000, 104, 9385 .

(36) Liang, N.; Miller, J. R.; Closs, G. L. J. Am. Chem. Soc. 1989, 111 8740. The negative slope of the reorganization energy vs temperature in a weakly polar solvent is obtained in this paper from the dielectric continuum estimate. The temperature correction of the solvent reorganization energy describes better the observed temperature dependence of the ET rate.

(37) Elliott, C. M.; Derr, D. L.; Matyushov, D. V.; Newton, M. D. J. Am. Chem. Soc. 1998, 120, 11714.

(38) Nelsen, S. F.; Ismagilov, R. F.; Gentile, K. E.; Powell, D. R. J. Am. Chem. Soc. 1999, 121, 7108.

(39) Derr, D. L.; Elliott, C. M. J. Phys. Chem. A 1999, 103, 7888.

(40) Zhao, X.; Burt, J. A.; Knorr, F. J.; McHale, J. L. J. Phys. Chem. A 2001, 105, 11110 .

(41) Wetzler, D. E.; Chesta, C.; Fernández-Prini, R.; Aramendía, P. F. J. Phys. Chem. A 2002, 106, 2390.

(42) Kawski, A.; Kikliński, B.; Bojarski, P. Chem. Phys. 2006, 320, 188.

(43) Dong, Y.; Hupp, J. T. Inorg. Chem. 1992, 31, 3322.

(44) Mertz, E. L. J. Phys. Chem. A 2005, 109, 44.

(45) Coropceanu, V.; Lambert, C.; Nöll, G.; Brédas, J. L. Chem. Phys. Lett. 2003, 373, 153.

(46) D’Alessandro, D. M.; Junk, P. C.; Keene, F. R. Supramolecular Chemistry 2005, 17, 529.

(47) Carter, E. A.; Hynes, J. T. J. Chem. Phys. 1991, 94, 5961.

(48) Straus, J. B.; Voth, G. A. J. Phys. Chem. 1993, 97, 7388.

(49) Marchi, M.; Gehlen, J. N.; Chandler, D.; Newton, M. J. Am. Chem. Soc. 1993, 115, 4178 . 419 . 
(51) Ando, K. J. Chem. Phys. 2001, 115, 5228.

(52) Kato, S.; Amatatsu, Y. J. Chem. Phys. 1990, 92, 7241.

(53) Ando, K. J. Chem. Phys. 1994, 101, 2850.

(54) Gray, C. G.; Gubbins, K. E. Theory of Molecular Liquids; Clarendon Press: Oxford, 1984

(55) Yu, H.-A.; Karplus, M. J. Chem. Phys. 1988, 89, 2366.

(56) Guillot, B.; Guissani, Y. J. Chem. Phys. 1993, 99, 8075.

(57) Ben-Amotz, D.; Raineri, F. O.; Stell, G. J. Phys. Chem. B 2005, $109,6866$.

(58) Lazaridis, T. J. Phys. Chem. B 2000, 104, 4964.

(59) Frisch, M. J.; Trucks, G. W.; Scuseria, G. E.; Robb, M. A.; Cheeseman, J. R.; Zakrzewski, V. G. GAUSSIAN 03; Pittsburgh, PA, 2003

(60) Donohue, J. Acta Crystallogr. 1956, 9, 960.

(61) Farztdinov, V. M.; Schanz, R.; Kovalenko, S. A.; Ernsting, N. P. J. Phys. Chem. A 2000, 104, 11486.

(62) Rashid, A. N. J. Mol. Struct. 2004, 681, 57.

(63) Jorgensen, W. L.; Maxwell, D. S.; Tirado-Rives, J. J. Am. Chem. Soc. 1996, 118,11225 .
(64) Allen, M. P.; Tildesley, D. J. Computer Simulation of Liquids; Clarendon Press: Oxford, 1996.

(65) Forester, T. R.; Smith, W. The DL-POLY-2.0 Reference Manual, version 2.0; CCLRC, Daresbury Laboratory: Warrington, England, 1985.

(66) Berendsen, H. J. C.; Grigera, J. R.; Straatsma, T. P. J. Phys. Chem. $1987,91,6269$.

(67) H. J. Boehm, R. M. Lynden-Bell, P. A. M.; McDonald, R. I. Mol. Phys. 1984, 51, 761.

(68) Ladanyi, B. M.; Perng, B.-C. J. Phys. Chem. B 2002, 106, 6922 (69) DeLeeuw, S. W.; Perram, J. W.; Smith, E. R. Proc. R. Soc. London, Ser. A 1980, 373, 27.

(70) Smith, W.; Forester, T. R. J. Mol. Graphics 1996, 14, 136141.

(71) Fonseca, T.; Ladanyi, B. M. J. Mol. Liq. 1994, 60, 1

(72) Geissler, P. L.; Chandler, D. J. Chem. Phys. 2000, 113, 9759.

(73) Zhou, H.-X.; Szabo, A. J. Chem. Phys. 1995, 103, 3481

(74) Marcus, Y. The Properties of Solvents; Wiley: Chichester, 1998. 